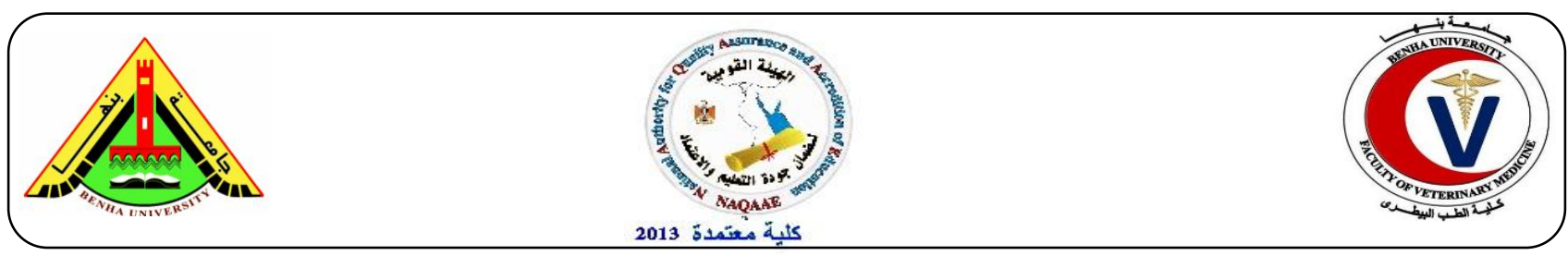

\title{
Influence of lactoferrin on immune response in rats intoxicated by diazinon
} Assaraj Q.SH ${ }^{1}$, Alattar H.A ${ }^{2}$, Farid A.S ${ }^{3}$, Fararah K.M ${ }^{3}$

${ }^{1}$ Department of internal medicine, college of veterinary, Bahry University, Sudan.

${ }^{2}$ Department of Animal Mediine, Faculty of Veterinary Medicine, Benha University

${ }^{3}$ Department of Clinical pathology, Faculty of Veterinary Medicine, Benha University

\section{A B S T R A C T}

This study is done to evaluate the antioxidant effect of Lactoferrin (LF) on liver injury induced by Diazinon (DZN). Seventy five rats were divided into five groups, each group contains 15 rats; control (G1): (no treatments), Lactoferrin (G2): (LF, $100 \mathrm{mg} / \mathrm{kg}$ bw orally daily for 6 weeks), diazinon (G3): (DZN, $70 \mathrm{mg} / \mathrm{kg}$ bw orally, daily for 4 weeks), DZN +LF (G4): (DZN, $70 \mathrm{mg} / \mathrm{kg}$ bw orally, daily for 4 weeks followed by LF, $100 \mathrm{mg} / \mathrm{kg}$ bw orally daily for 2 weeks, and (LF $+\mathrm{DZN}$ ) group (G5): (LF, $100 \mathrm{mg} / \mathrm{kg}$ bw orally daily for seven weeks and DZN, $70 \mathrm{mg} / \mathrm{kg}$ bw for four weeks only started at the second week of the experiment).Total leukocyte count (TLC) and differential leukocyte count (DLC) were checked in whole blood, cytokine IL-10, total proteins(TP) and protein electrophoresis were checked in serum. In comparison to control group, WBCs found increased significantly in DZN group $(P<0.005)$ and IL-10, TP, albumin, and globulin were found significantly decreased too. In comparison to DZN group, WBCs and IL-10, levels were found significantly increased in $(\mathrm{DZN}+\mathrm{LF})$ group $(\mathrm{P}<0.05), \mathrm{TP}$, albumin, and globulin levels were also found significantly increased $(\mathrm{P}<0.05)$, WBCs found significantly decreased in $(\mathrm{LF}+\mathrm{DZN})$ group and IL-10, TP, albumin, and globulin were found significantly increased too $(\mathrm{P}<0.05)$.

Keywords: Lactoferrin, rat, Liver, Immune response, diazinon.

(http://www.bvmi.bu.edu.eg)

(BVMJ-34(2): 169-181, 2018)

\section{INTRODUCTION}

Lactoferrin (LF), a member of the transferrin family, strongly binds two ferric ions, plays notable roles in host defense against infections, immunomodulation, prevention from oxidative stress by binding pro-oxidative iron ions, cell growth regulation and inhibition of binding of lipopolysaccharides to bioactive cellular molecules (Haversen et al., 2002, Farnaud and Evans, 2003). Immune modulatory actions of LF are mediated by modulation of cytokine production, since LF has been shown to decrease TNF- $\alpha$, IL-1 $\beta$ and IL-6 production in vitro in human mononuclear cells, (Crouch et al., 1992) and in vivo in mice (Zimecki et al., 1998 and Kruzel et al., 2002).

The increase of serum cytokine and acute phase protein occur early in disease progress even before the observation of clinical signs, are in correlation with more individual assessment of pain and distress. Considerable acute- phase proteins migrate in $\alpha, \beta$, and $\gamma$ fractions. Thus progressive variation in serum albumin and globulin levels may provide 
early and worthy diagnostic information and prognosis in dealing with clinical conditions of animals. In recent studies done by Yen et al., (2011)a high dose of pLF led to significant increases in serum $\operatorname{IgA}, \operatorname{IgG}$ and IBD-specific anti-bodies.

Diazinon (DZN) is a worldwide organophosphorus (OP) insecticide, used in many different fields concerning agriculture and domestic uses (Garfitt et al., 2002). It was puzzle out in Recent studies that acute and chronic toxicity of organophosphorus insecticides (OPIs) depends on induction of oxidative stress through generation of free radicals or reactive oxygen species (ROS) and adversely affect on antioxidants scavenging enzymes in mammals and other organisms in different tissues (Ogutcu et al., 2006), that according to Hilal et al., (2015) DZN induces significant liver damage.

\section{Materials and methods}

\subsection{Experimental animals:}

Seventy five male Wistar rats weighing about 140-180 gm were obtained from the Animal House, Faculty of Veterinary Medicine, Benha University, Egypt. All animals were caged and maintained on a standard pellets diet, with free access to tap water and were acclimatized for one week before fulfillment of the experiment. Body weights of experimental rats in all the groups were weekly estimated to adjust the dose of applied chemicals using an electronic balance.

\subsection{Chemicals:}

Diazinon (DZN)

Diazinon $60 \%$ (Emulsifiable concentrate) containing $60 \%$ active ingredient was obtained from High Control Company, Cairo, Egypt. It was dissolved in distilled water to obtain the required dose concentration (70mg/Kg bw). (El-Shenawy et al., 2010)

Bovine Lactoferrin $(L F)$
Bovine Lactoferrin (LF) was provided by Jarrow Formulas (Superior Nutrition and Formulation, Los Angeles). LF was prepared to give a required dose concentration of (100mg/Kg bw) by dissolving LF in distilled water. (Aytül et al., 2010)

\subsection{Experimental design:}

The design is shown in table 1.

\subsection{Blood samples:}

Blood samples were taken from each animal under light ether anesthesia by puncturing the retro-orbital venous plexus of the animal with a fine sterilized glass capillary tube. The samples were divided into two parts: Samples for the WBC and DLC, were collected in EDTA tubes and evaluated by using Automatic cell counter (H.A-Vet clindiage, Belgium) which depend on both electrical and optical techniques according to (Knapp et al., 1996), and samples for determination of total proteins, serum protein electrophoresis, and Cytokines (IL-10) were collected in a plain tubes; the blood was allowed to stand at room temperature for at least 30 minutes then centrifuged at 3000 relative centrifugal force (rcf) for 15 minutes. The serum was collected in sealed $1.5 \mathrm{ml}$ Eppendorf tube and stored in deep freeze $\left(-20^{\circ} \mathrm{C}\right)$.

Serum total proteins were determined by the reaction described by Weichselbaum (1946). Serum protein electrophoresis was performed according to the method described by Keyser and Watkins, (1972).

Samples were taken after $4^{\text {th }}, 5^{\text {th }}$, and $6^{\text {th }}$ weeks post DZN administration.

\subsection{Statistical analysis:}

Data were analyzed using SPSS 11.0 for windows. The significance was calculated using one-way analysis of variance (ANOVA) and followed by Tukey multiple comparison procedure to calculate the significance. $\mathrm{P}<$ 0.05 value was taken as statistically significant. 


\section{RESULTS}

At the first collection ( $5^{\text {th }}$ week), In comparison to control group (group 1) rats treated with LF (group 2) showed significant increase in total WBCs, accompanied by significant increase in IL-10 and significant increase in globulin, alpha1, alpha2, beta, and gamma globulin, and significant decrease in A/G ratio and no significant change in total protein and albumin. Rats intoxicated with DZN (group 3) showed significant increase in total WBCs granulocytes, lymphocytes, and monocytes, significant decrease in IL-10 concentration, significant decrease in total protein, globulin, beta, and gamma globulin, significant increase in $\mathrm{A} / \mathrm{G}$ ratio, and no significant change in albumin, alpha1, alpha2in comparison to control group (group $1)$.

In comparison to DZN group (group 3) rats treated with LF after intoxication with DZN (group 4) showed no significant change in leukocytes and differential leukocytes counts, and also no significant change in IL-10 concentration, total protein, and protein electrophoresis estimations. Rats in (group 5) showed significant decrease in total WBCs and granulocytes, and revealed no significant change in lymphocytes and monocytes, accompanied by significant increase in IL-10 and significant decrease in alpha1, and beta globulin, significant increase in total protein, albumin, gamma globulin, and no significant change in, globulin, A/G ratio, and alpha2

At the second collection $\left(6^{\text {th }}\right.$ week) in comparison to control group (group 1), data of rats treated with LF (group 2) showed significant increase in total WBCs, granulocytes, and monocytes, accompanied by significant increase in IL-10 concentration and significant increase in total protein, alpha1, and gamma globulin, and no significant change in, albumin, globulin, A/G ratio, alpha2, and beta globulin. Rats intoxicated with DZN (group 3) showed significant increase in total WBCs, lymphocytes, granulocytes, and monocytes, significant decrease in IL-10 concentration, significant decrease in total protein, globulin, alpha1, alpha2, beta and gamma globulin, and no significant change in albumin. In comparison to DZN group (group 3) rats treated with LF after intoxication with DZN (group 4) showed significant increase in total WBCs and granulocytes, and significant decrease in lymphocytes, accompanied by significant increase in IL-10 concentration and significant increase in total protein, albumin, globulin, alpha1, beta, and gamma globulin and no significant change in $A / G$ ratio, alpha2.Rats treated with $\mathrm{LF}$ as prevention to toxicity of DZN (group 5) showed significant decrease in total WBCs, lymphocytes and monocytes but no significant change in granulocytes, accompanied by significant increase in IL-10 concentration and significant increase in total protein, albumin, globulin, A/G ratio, alpha1, alpha2, and gamma globulin, and no significant increase in beta.

At the third collection ( $7^{\text {th }}$ week), in comparison to control group, rats treated with LF (group 2) showed significant increase in total WBCs, and no significant change in granulocytes, lymphocytes, and monocytes, significant increase in IL-10 concentration and significant increase in albumin, A/G ratio, alpha1, significant decrease in globulin, alpha2, beta, and gamma globulin, and no significant change in total protein. Rats intoxicated with DZN (group 3) showed significant increase in total WBCs, and no significant change in granulocytes, lymphocytes, and monocytes, accompanied by significant decrease in IL-10 concentration, significant decrease in total protein, albumin, globulin, alpha1, alpha2, 
beta and gamma globulin, and no significant change in $\mathrm{A} / \mathrm{G}$ ratio.

In comparison to diazinon group (group 3) rats treated with LF after intoxication with DZN (group 4) showed significant decrease in total WBCs and no significant change in granulocytes, lymphocytes, and monocytes, significant increase in IL-10 and significant increase in total protein, albumin, globulin, $\mathrm{A} / \mathrm{G}$ ratio, alpha1, beta and gamma globulin, and no significant change in alpha2.Rats treated with LF as prevention to toxicity of DZN (group 5) showed significant decrease in total WBCs and no significant change in granulocytes, lymphocytes and monocytes, significant increase in IL-10 and significant increase in total protein, albumin, globulin, $\mathrm{A} / \mathrm{G}$ ratio, beta, and gamma globulin, and significant decrease in alpha1, alpha2.

Table 1: The design of the experiment.

\begin{tabular}{|c|c|c|c|c|}
\hline Groups & $\begin{array}{l}\text { No of } \\
\text { animal } \\
\text { /group }\end{array}$ & Treatments & $\begin{array}{l}\text { Type and } \\
\text { time } \\
\text { of sampling }\end{array}$ & Parameters \\
\hline Control & 15 & $\begin{array}{llll}\begin{array}{l}\text { Normal saline } \\
\text { weeks }\end{array} & & & \\
\end{array}$ & \multirow{5}{*}{ 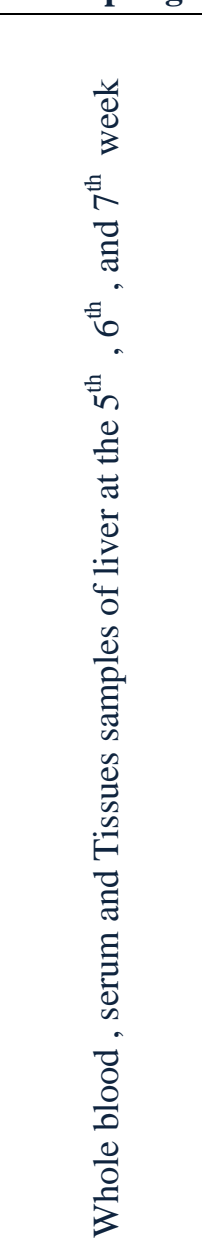 } & \multirow{5}{*}{$\begin{array}{l}\text {-CBC } \\
\text {-T. protein } \\
\text {-Protein } \\
\text { electrophoresis } \\
\text {-IL-10 }\end{array}$} \\
\hline Diazinon & 15 & $\begin{array}{l}\text { DZN, } 70 \mathrm{mg} / \mathrm{kg} \text { body } \\
\text { weight orally in distilled } \\
\text { water daily for } 4 \text { weeks } \\
\left.\text { ( } 2^{\text {nd }} \text { to } 5^{\text {th }} \text { week }\right) \text {. }\end{array}$ & & \\
\hline Lactoferrin & 15 & $\begin{array}{l}\text { LF } 100 \mathrm{mg} / \mathrm{kg} \text { orally } \\
\text { daily for } 6 \text { weeks }\left(2^{\text {nd }} \text { to }\right. \\
\left.7^{\text {th }} \text { week }\right) .\end{array}$ & & \\
\hline $\mathrm{DZN+LF}$ & 15 & $\begin{array}{l}\text { DZN, } 70 \mathrm{mg} / \mathrm{kg} \text { body } \\
\text { weight orally in distilled } \\
\text { water daily for } 4 \text { weeks } \\
\left(2^{\text {nd }} \text { to } 5^{\text {th }} \text { weeks }\right) \text {. } \\
\text { Lactoferin } 100 \mathrm{mg} / \mathrm{kg} \\
\text { orally daily for } 2 \text { weeks } \\
\left(6^{\text {th }} \& 7^{\text {th }} \text { weeks }\right)\end{array}$ & & \\
\hline $\mathbf{L F}+\mathrm{DZN}$ & 15 & $\begin{array}{l}\text { LF } 100 \mathrm{mg} / \mathrm{kg} \text { orally; } \\
\text { daily for the } 7 \text { weeks. } \\
\text { DZN } 70 \mathrm{mg} / \mathrm{kg} \text { body } \\
\text { weight orally for } 4 \\
\text { weeks }\left(2^{\text {nd }} \text { to } 5^{\text {th }}\right. \\
\text { weeks). }\end{array}$ & & \\
\hline
\end{tabular}


Table 2: Leukogram of control, LF, DZN, DZN+LF, and LF+ DZN groups at 4 weeks post DZN administration.

\begin{tabular}{|c|c|c|c|c|}
\hline Groups & $\begin{array}{l}\text { WBC } \\
\left(\times 10^{3} / \mu \mathrm{l}\right)\end{array}$ & $\begin{array}{l}\text { GRAN } \\
\left(\times 10^{3} / \mu 1\right)\end{array}$ & $\begin{array}{l}\text { LYMPH } \\
\left(\times 10^{3} / \mu 1\right)\end{array}$ & $\begin{array}{l}\text { MONO } \\
\left(\times 10^{3} / \mu 1\right)\end{array}$ \\
\hline G1 (Control) & $\begin{array}{l}11.57 \\
\pm 0.49^{c}\end{array}$ & $\begin{array}{l}3.38 \\
\pm 0.1^{\mathbf{b}}\end{array}$ & $\begin{array}{l}5.86 \\
\pm 0.66^{\mathbf{b}}\end{array}$ & $\begin{array}{l}1.09 \\
\pm 0.08^{\mathbf{b}}\end{array}$ \\
\hline G2(Lactoferrin) & $\begin{array}{l}12.90 \\
\pm 0.35^{b}\end{array}$ & $\begin{array}{l}4.16 \\
\pm 0.06^{b}\end{array}$ & $\begin{array}{l}6.68 \\
\pm 0.19^{b}\end{array}$ & $\begin{array}{l}1.05 \\
\pm 0.02^{\mathbf{b}}\end{array}$ \\
\hline G3 (Diazinon) & $\begin{array}{l}14.87 \\
\pm 0.49^{\mathrm{a}}\end{array}$ & $\begin{array}{l}6.44 \\
\pm 0.54^{\mathrm{a}}\end{array}$ & $\begin{array}{l}8.36 \\
\pm 0.47^{\mathrm{a}}\end{array}$ & $\begin{array}{l}1.31 \\
\pm 0.26^{\mathrm{a}}\end{array}$ \\
\hline G4 (DZN+LF) & $\begin{array}{l}14.87 \\
\pm 0.49^{\mathrm{a}}\end{array}$ & $\begin{array}{l}6.44 \\
\pm 0.54^{\mathrm{a}}\end{array}$ & $\begin{array}{l}8.36 \\
\pm 0.47^{\mathrm{a}}\end{array}$ & $\begin{array}{l}1.31 \\
\pm 0.26^{\mathrm{a}}\end{array}$ \\
\hline G5 (LF+DZN) & $\begin{array}{l}12.43 \\
\pm 0.91^{c}\end{array}$ & $\begin{array}{l}4.64 \\
\pm 0.03^{b}\end{array}$ & $\begin{array}{l}8.03 \\
\pm 0.61^{\mathrm{a}}\end{array}$ & $\begin{array}{l}1.50 \\
\pm 0.03^{\mathrm{a}}\end{array}$ \\
\hline
\end{tabular}

Results are expressed as mean \pm S. E. M.

Different subscripts (a, b, c, d, e) at the same column indicate significant differences at $\mathrm{P}>0.05$

Table 3: Leukogram of control, LF, DZN, DZN+LF, and LF+ DZN groups at 5 weeks post DZN administration.

\begin{tabular}{lllll}
\hline Groups & $\begin{array}{l}\text { WBC } \\
\left(\times 10^{3} / \mu \mathrm{l}\right)\end{array}$ & $\begin{array}{l}\text { GRAN } \\
\left(\times 10^{3} / \mu \mathrm{l}\right)\end{array}$ & $\begin{array}{l}\text { LYMPH } \\
\left(\times 10^{3} / \mu \mathrm{l}\right)\end{array}$ & $\begin{array}{l}\text { MONO } \\
\left(\times 10^{3} / \mu \mathrm{l}\right)\end{array}$ \\
\hline G1 (Control) & 11.72 & 3.38 & 6.30 & 1.02 \\
& $\pm 0.62^{\mathbf{d}}$ & $\pm 0.13^{\mathbf{b}}$ & $\pm 0.63^{\mathbf{b}}$ & $\pm 0.09^{\mathbf{c}}$ \\
G2(Lactoferrin) & 14.93 & 3.71 & 6.19 & 1.61 \\
& $\pm 0.41^{\mathbf{b}}$ & $\pm 0.23^{\mathbf{b}}$ & $\pm 0.31^{\mathbf{b}}$ & $\pm 0.03^{\mathbf{a}}$ \\
G3 (Diazinon) & $13.5^{\mathbf{b}}$ & $4.81^{\mathbf{b}}$ & 8.67 & 1.23 \\
& $\pm 0.20^{\mathbf{c}}$ & $\pm 0.34^{\mathbf{b}}$ & $\pm 0.79^{\mathbf{a}}$ & $\pm 0.04^{\mathbf{b}}$ \\
G4 (DZN+LF) & $18.33^{\mathbf{a}}$ & $8.9^{\mathbf{a}}$ & 6.76 & 1.28 \\
& $\pm 1.31^{\mathbf{a}}$ & $\pm 0.81^{\mathbf{a}}$ & $\pm 0.74^{\mathbf{b}}$ & $\pm 0.15^{\mathbf{b}}$ \\
G5 (LF+DZN) & 11.53 & $2.64^{\mathbf{b}}$ & 6.65 & $1.01^{\mathbf{c}}$ \\
& $\pm 0.26^{\mathbf{d}}$ & $\pm 0.59^{\mathbf{b}}$ & $\pm 0.56^{\mathbf{b}}$ & $\pm 0.10^{\mathbf{c}}$ \\
\hline
\end{tabular}

Results are expressed as mean \pm S. E. M.

Different subscripts (a, b, c, d, e) at the same column indicate significant differences at $\mathrm{P}>0.05$ 
Table 4: Leukogram of control, LF, DZN, DZN+LF, and LF+ DZN groups at 6 weekspost DZN administration.

\begin{tabular}{|c|c|c|c|c|}
\hline Groups & $\begin{array}{l}\text { WBC } \\
\left(\times 10^{3} / \mu 1\right)\end{array}$ & $\begin{array}{l}\text { GRAN } \\
\left(\times 10^{3} / \mu 1\right)\end{array}$ & $\begin{array}{l}\text { LYMPH } \\
\left(\times 10^{3} / \mu 1\right)\end{array}$ & $\begin{array}{l}\text { MONO } \\
\left(\times 10^{3} / \mu \mathrm{l}\right)\end{array}$ \\
\hline G1 (Control) & $\begin{array}{l}11.27 \\
\pm 0.94^{\mathrm{c}}\end{array}$ & $\begin{array}{l}1.81 \\
\pm 0.15^{\mathrm{a}}\end{array}$ & $\begin{array}{l}9.67 \\
\pm 0.40^{\mathrm{a}}\end{array}$ & $\begin{array}{l}1.12 \\
\pm 0.11^{\mathrm{a}}\end{array}$ \\
\hline G2(Lactoferrin) & $\begin{array}{l}13.11 \\
\pm 0.60^{b}\end{array}$ & $\begin{array}{l}2.71 \\
\pm 0.03^{a}\end{array}$ & $9.62 .44^{\mathrm{a}}$ & $\begin{array}{l}1.28 \\
\pm 0.05^{\mathrm{a}}\end{array}$ \\
\hline G3 (Diazinon) & $\begin{array}{l}15.87 \\
\pm 0.03^{\mathrm{a}}\end{array}$ & $\begin{array}{l}2.77 \\
\pm 0.06^{\mathrm{a}}\end{array}$ & $\begin{array}{l}10.03 \\
\pm 0.75^{\mathrm{a}}\end{array}$ & $\begin{array}{l}1.34 \\
\pm 0.09^{\mathrm{a}}\end{array}$ \\
\hline G4 (DZN+LF) & $\begin{array}{l}11.36 \\
\pm 0.32^{c}\end{array}$ & $\begin{array}{l}1.23 \\
\pm 0.22^{\mathrm{a}}\end{array}$ & $\begin{array}{l}8.96 \\
\pm 0.75^{\mathrm{a}}\end{array}$ & $\begin{array}{l}1.17 \\
\pm 0.16^{\mathrm{a}}\end{array}$ \\
\hline G5 (LF+DZN) & $\begin{array}{l}10.23 \\
\pm 0.29^{d}\end{array}$ & $\begin{array}{l}1.43 \\
\pm 0.33^{\mathrm{a}}\end{array}$ & $\begin{array}{l}9.62 \\
\pm 0.76^{\mathrm{a}}\end{array}$ & $\begin{array}{l}1.25 \\
\pm 0.13^{\mathrm{a}}\end{array}$ \\
\hline
\end{tabular}

Table 5: Protein electrophoresis in control, LF, DZN, DZN+LF, and LF+ DZN groups at 4th weekspost DZN administration.

\begin{tabular}{|c|c|c|c|c|c|}
\hline $\begin{array}{l}\text { Groups } \\
\text { parameter } \\
\text { s }\end{array}$ & Control & $\begin{array}{l}\text { Lactoferrin } \\
\text { (LF) }\end{array}$ & $\begin{array}{l}\text { Diazinon } \\
(\mathrm{DZN})\end{array}$ & $\mathrm{DZN}+\mathrm{LF}$ & $\mathrm{LF}+\mathrm{DZN}$ \\
\hline $\begin{array}{l}\text { T. protein } \\
(\mathrm{g} / \mathrm{dL})\end{array}$ & $\begin{array}{l}7.44 \\
\pm 0.22^{\mathrm{a}}\end{array}$ & $\begin{array}{l}7.60 \\
\pm 0.08^{\mathrm{a}}\end{array}$ & $\begin{array}{l}6.91 \\
\pm 0.07^{\mathrm{c}}\end{array}$ & $\begin{array}{l}6.91 \\
\pm 0.07^{\mathrm{c}}\end{array}$ & $\begin{array}{l}7.26 \\
\pm 0.12^{b}\end{array}$ \\
\hline $\begin{array}{l}\text { Albumin } \\
\text { (g/dl) }\end{array}$ & $\begin{array}{l}2.97 \\
\pm 0.07^{\mathrm{a}}\end{array}$ & $\begin{array}{l}3.00 \\
\pm 0.17^{\mathrm{a}}\end{array}$ & $\begin{array}{l}3.07 \\
\pm 0.03^{\mathrm{a}}\end{array}$ & $\begin{array}{l}3.07 \\
\pm 0.03^{\mathrm{a}}\end{array}$ & $\begin{array}{l}2.93 \\
\pm 0.03^{\mathrm{b}}\end{array}$ \\
\hline $\begin{array}{l}\text { Globulin } \\
(\mathrm{g} / \mathrm{dl})\end{array}$ & $\begin{array}{l}4.27 \\
\pm 0.19^{\mathrm{b}}\end{array}$ & $\begin{array}{l}4.87 \\
\pm 0.06^{\mathrm{a}}\end{array}$ & $\begin{array}{l}3.90 \\
\pm 0.06^{\mathrm{c}}\end{array}$ & $\begin{array}{l}3.90 \\
\pm 0.06^{\mathrm{c}}\end{array}$ & $\begin{array}{l}3.93 \\
\pm 0.15^{\mathrm{c}}\end{array}$ \\
\hline $\mathrm{A} / \mathrm{G}$ ratio & $\begin{array}{l}0.73 \\
\pm 0.03^{\mathrm{a}}\end{array}$ & $\begin{array}{l}0.63 \\
\pm 0.03^{\mathrm{c}}\end{array}$ & $\begin{array}{l}0.80 \\
\pm 0.02^{\mathrm{a}}\end{array}$ & $\begin{array}{l}0.80 \\
\pm 0.02^{\mathrm{a}}\end{array}$ & $\begin{array}{l}0.77 \\
\pm 0.03^{\mathrm{a}}\end{array}$ \\
\hline $\begin{array}{l}\text { Alpha } 1 \\
(\mathrm{~g} / \mathrm{dl})\end{array}$ & $\begin{array}{l}1.07 \\
\pm 0.06^{\mathrm{b}}\end{array}$ & $\begin{array}{l}1.17 \\
\pm 0.03^{\mathrm{a}}\end{array}$ & $\begin{array}{l}1.13 \\
\pm 0.03^{\mathrm{a}}\end{array}$ & $\begin{array}{l}1.13 \\
\pm 0.03^{\mathrm{a}}\end{array}$ & $\begin{array}{l}0.80 \\
\pm 0.03^{c}\end{array}$ \\
\hline $\begin{array}{l}\text { Alpha } 2 \\
(\mathrm{~g} / \mathrm{dl})\end{array}$ & $\begin{array}{l}1.00 \\
\pm 0.06^{\mathrm{b}}\end{array}$ & $\begin{array}{l}1.20 \\
\pm 0.03^{\mathrm{a}}\end{array}$ & $\begin{array}{l}1.00 \\
\pm 0.06^{\mathrm{b}}\end{array}$ & $\begin{array}{l}1.00 \\
\pm 0.06^{\mathrm{b}}\end{array}$ & $\begin{array}{l}0.93 \\
\pm 0.07^{\mathrm{b}}\end{array}$ \\
\hline $\begin{array}{l}\text { Beta } \\
(\mathrm{g} / \mathrm{dl})\end{array}$ & $\begin{array}{l}1.10 \\
\pm 0.06^{\mathrm{b}}\end{array}$ & $\begin{array}{l}1.17 \\
\pm 0.07^{\mathrm{a}}\end{array}$ & $\begin{array}{l}0.93 \\
\pm 0.03^{\mathrm{c}}\end{array}$ & $\begin{array}{l}0.93 \\
\pm 0.03^{\mathrm{c}}\end{array}$ & $\begin{array}{l}0.77 \\
\pm 0.03^{\mathrm{d}}\end{array}$ \\
\hline $\begin{array}{l}\text { Gamma } \\
(\mathrm{g} / \mathrm{dl})\end{array}$ & $\begin{array}{l}1.03 \\
\pm 0.07^{\mathrm{c}}\end{array}$ & $\begin{array}{l}1.27 \\
\pm 0.07^{\mathrm{a}}\end{array}$ & $\begin{array}{l}0.77 \\
\pm 0.03^{\mathrm{d}}\end{array}$ & $\begin{array}{l}0.77 \\
\pm 0.03^{\mathrm{d}}\end{array}$ & $\begin{array}{l}1.20 \\
\pm 0.03^{\mathrm{b}}\end{array}$ \\
\hline
\end{tabular}


Table 6: Protein electrophoresis in control, LF, DZN, DZN+LF, and LF+ DZN groups at 5th weekspost DZN administration.

\begin{tabular}{llllll}
\hline $\begin{array}{l}\text { Groups } \\
\text { parameter }\end{array}$ & Control & $\begin{array}{l}\text { Lactoferrin } \\
(\mathrm{LF})\end{array}$ & $\begin{array}{l}\text { Diazinon } \\
(\mathrm{DZN})\end{array}$ & DZN+LF & LF+DZN \\
$\mathrm{S}$ & & & & \\
\hline T. protein & 7.37 & 7.60 & 6.56 & 6.86 & 7.26 \\
(g/dL) & $\pm 0.12^{\mathrm{b}}$ & $\pm 0.10^{\mathrm{a}}$ & $\pm 0.08^{\mathrm{d}}$ & $\pm 0.27^{\mathrm{c}}$ & $\pm 0.16^{\mathrm{b}}$ \\
Albumin & 2.97 & 2.90 & 2.90 & 3.20 & 3.40 \\
(g/dl) & $\pm 0.07^{\mathrm{c}}$ & $\pm 0.10^{\mathrm{c}}$ & $\pm 0.15^{\mathrm{c}}$ & $\pm 0.09^{\mathrm{b}}$ & $\pm 0.25^{\mathrm{a}}$ \\
Globulin & 4.27 & 4.27 & 3.50 & 3.67 & 4.37 \\
(g/dl) & $\pm 0.19^{\mathrm{a}}$ & $\pm 0.15^{\mathrm{a}}$ & $\pm 0.09^{\mathrm{c}}$ & $\pm 0.20^{\mathrm{b}}$ & $\pm 0.10^{\mathrm{a}}$ \\
A/G ratio & 0.73 & 0.73 & $0.87^{\mathrm{a}}$ & 0.83 & 0.77 \\
& $\pm 0.03^{\mathrm{a}}$ & $\pm 0.05^{\mathrm{a}}$ & $\pm 0.05^{\mathrm{a}}$ & $\pm 0.03^{\mathrm{a}}$ & $\pm 0.09^{\mathrm{a}}$ \\
Alpha 1 & 1.07 & 1.20 & 0.80 & 0.90 & 1.05 \\
(g/dl) & $\pm 0.06^{\mathrm{b}}$ & $\pm 0.06^{\mathrm{a}}$ & $\pm 0.03^{\mathrm{d}}$ & $\pm 0.03^{\mathrm{c}}$ & $\pm 0.03^{\mathrm{b}}$ \\
Alpha 2 & 1.00 & 1.03 & 0.80 & 0.70 & 1.00 \\
(g/dl) & $\pm 0.06^{\mathrm{a}}$ & $\pm 0.03^{\mathrm{a}}$ & $\pm 0.00^{\mathrm{b}}$ & $\pm 0.00^{\mathrm{b}}$ & $\pm 0.06^{\mathrm{a}}$ \\
Beta & 1.10 & 1.07 & $0.87^{\mathrm{b}}$ & 1.20 & 1.20 \\
(g/dl) & $\pm 0.06^{\mathrm{b}}$ & $\pm 0.03^{\mathrm{b}}$ & $\pm 0.06^{\mathrm{c}}$ & $\pm 0.06^{\mathrm{a}}$ & $\pm 0.03^{\mathrm{a}}$ \\
Gamma & 1.03 & 1.10 & 0.93 & 0.87 & 1.03 \\
(g/dl) & $\pm 0.07^{\mathrm{b}}$ & $\pm 0.00^{\mathrm{a}}$ & $\pm 0.07^{\mathrm{c}}$ & $\pm 0.03^{\mathrm{d}}$ & $\pm 0.03^{\mathrm{b}}$ \\
\hline
\end{tabular}

Table 7: Protein electrophoresis in control, LF, DZN, DZN+LF, and LF+ DZN groups at 6th weekspost DZN administration.

\begin{tabular}{llllll}
\hline $\begin{array}{l}\text { Groups } \\
\text { parameters }\end{array}$ & Control & $\begin{array}{l}\text { Lactoferrin } \\
(\mathrm{LF})\end{array}$ & $\begin{array}{l}\text { Diazinon } \\
(\mathrm{DZN})\end{array}$ & DZN+LF & LF+DZN \\
\hline T. protein & 7.06 & 6.97 & 6.15 & 6.68 & 6.87 \\
$(\mathrm{~g} / \mathrm{dL})$ & $\pm 0.22^{\mathrm{a}}$ & $\pm 0.21^{\mathrm{a}}$ & $\pm 0.10^{\mathrm{c}}$ & $\pm 0.12^{\mathrm{b}}$ & $\pm 0.05^{\mathrm{a}}$ \\
Albumin & 2.80 & 3.17 & 2.40 & 2.97 & 3.23 \\
$(\mathrm{~g} / \mathrm{dl})$ & $\pm 0.06^{\mathrm{c}}$ & $\pm 0.13^{\mathrm{a}}$ & $\pm 0.03^{\mathrm{d}}$ & $\pm 0.00^{\mathrm{b}}$ & $\pm 0.12^{\mathrm{a}}$ \\
Globulin & 3.87 & 3.67 & $3.57^{\mathrm{a}}$ & 3.83 & 3.27 \\
(g/dl) & $\pm 0.18^{\mathrm{a}}$ & $\pm 0.09^{\mathrm{b}}$ & $\pm 0.09^{\mathrm{b}}$ & $\pm 0.18^{\mathrm{a}}$ & $\pm 0.09^{\mathrm{c}}$ \\
A/G ratio & 0.73 & 0.87 & 0.70 & 0.77 & 1.00 \\
& $\pm 0.02^{\mathrm{b}}$ & $\pm 0.02^{\mathrm{a}}$ & $\pm 0.01^{\mathrm{b}}$ & $\pm 0.06^{\mathrm{b}}$ & $\pm 0.05^{\mathrm{a}}$ \\
Alpha 1 & 0.80 & 1.03 & $0.97^{\mathrm{b}}$ & 1.07 & 0.80 \\
(g/dl) & $\pm 0.06^{\mathrm{c}}$ & $\pm 0.00^{\mathrm{a}}$ & $\pm 0.03^{\mathrm{b}}$ & $\pm 0.06^{\mathrm{a}}$ & $\pm 0.03^{\mathrm{c}}$
\end{tabular}




$\begin{array}{llllll}\text { Alpha } 2 & 1.03 & 0.87 & 0.87 & 0.90 & 0.67 \\ (\mathrm{~g} / \mathrm{dl}) & \pm 0.04^{\mathrm{a}} & \pm 0.00^{\mathrm{b}} & \pm 0.06^{\mathrm{b}} & \pm 0.03^{\mathrm{b}} & \pm 0.07^{\mathrm{c}} \\ \text { Beta } & 1.07 & 0.97 & 0.70 & 1.00 & 1.00 \\ (\mathrm{~g} / \mathrm{dl}) & \pm 0.03^{\mathrm{a}} & \pm 0.03^{\mathrm{b}} & \pm 0.06^{\mathrm{c}} & \pm 0.06^{\mathrm{b}} & \pm 0.06^{\mathrm{b}} \\ \text { Gamma } & 0.97 & 0.83 & 0.83 & 0.83 & 0.90 \\ (\mathrm{~g} / \mathrm{dl}) & \pm 0.03^{\mathrm{a}} & \pm 0.00^{\mathrm{c}} & \pm 0.03^{\mathrm{c}} & \pm 0.06^{\mathrm{c}} & \pm 0.03^{\mathrm{b}}\end{array}$

Table 8: Changes of anti-inflammatory IL-10 (pg/ml) (mean \pm SE) in control, LF, DZN, DZN+LF, and LF+ DZN groups at 4, 5, and 6 weeks post DZN administration.

\section{PERIOD S}

\begin{tabular}{llll} 
GROUPS & First & Second & Third \\
\hline Control & 150.00 & 211.67 & 238.75 \\
& $\pm 10.11^{\mathbf{b}}$ & $\pm 2.89^{\mathbf{c}}$ & $\pm 10.00^{\mathbf{b}}$ \\
LF & 233.33 & 275.00 & 268.75 \\
& $\pm 7.22^{\mathbf{a}}$ & $\pm 11.55^{\mathbf{a}}$ & $\pm 13.64^{\mathbf{a}}$ \\
DZN & 117.50 & 125.00 & 205.00 \\
& $\pm 14.43^{\mathbf{c}}$ & $\pm 5.77^{\mathbf{e}}$ & $\pm 18.78^{\mathbf{d}}$ \\
DZN+LF & 117.50 & 158.33 & 233.00 \\
& $\pm 14.43^{\mathbf{c}}$ & $\pm 3.33^{\mathbf{d}}$ & $\pm 14.43^{\mathbf{c}}$ \\
LF+DZN & 233.33 & 258.33 & 250.00 \\
& $\pm 6.67^{\mathbf{a}}$ & $\pm 7.64^{\mathbf{b}}$ & $\pm 8.82^{\mathbf{b}}$ \\
\hline
\end{tabular}

\section{DISCUSSION}

Diazinon causes toxic effects on blood cells, spleen thymus and lymph nodes of rats, this may disturb the immune system, according to Hassan et al., (1988) Some OP insecticides causes increase and others causes decrease of WBC; he recorded an increase of WBC in dimethoate-treated male rabbits, while Garg et al., (2004) recorded a decrease of WBCs observed in monocrotophos-treated chickens.

In the present study diazinon caused significant increase in WBC as it appears in the leukogram vision; a significant increase in total WBCs, granulocytes, lymphocytes, and monocytes at the first and second collections $\left(5^{\text {th }}\right.$ and $6^{\text {th }}$ week). These findings were in accordance with findings of a study done by Kalender, et al., (2005) who recorded an increase in WBCs caused by DZN in experimental animals. This increase of WBCs could be resulted from the activation of defense mechanism of the animal's immunity system, caused by tissue damage and toxicity induced by DZN, this explanation also agrees with Kalender et al., (2006) who stated that the increase in leukocyte counts is an indication to activated animal's defense mechanism and immune system. And it could be also due to a tissue damage and necrosis caused by the pesticide.

Findings of leukocogram view of the three groups received bovine LF were 
variable between increase and decrease among the three groups and through the three collections $\left(5^{\text {th }}, 6^{\text {th }}\right.$, and $7^{\text {th }}$ weeks $)$; rats treated with LF (group 2) showed significant increase in total WBCs at the three collections $\left(5^{\text {th }}, 6^{\text {th }}\right.$, and $7^{\text {th }}$ weeks $)$ accompanied by a significant increase in granulocytes and monocytes at the second collection ( $6^{\text {th }}$ week), while rats treated with $\mathrm{LF}$ after intoxication with DZN (group 4) showed significant increase in total WBCs and granulocytes, and significant decrease in lymphocytes at the second collection $\left(6^{\text {th }}\right.$ week), rats treated with LF as prevention to toxicity of DZN showed significant decrease in total WBCs and granulocytes at the first collection $\left(5^{\text {th }}\right.$ week), and significant decrease in total WBCs lymphocytes, and monocytes at the second collection $\left(6^{\text {th }}\right.$ week) when compared with group 3 (DZN group).

This result of the leukogram picture demonstrate the regulatory role of LF in immunity system against the destructive effect to leukocytes by DZN, which agrees with Ward and Conneely (2004) they reported that the Up and down regulation of the immune system of LF in vivo by evidence for regulatory role of LF in the immune system and agrees also with Zimecki et al., (2001) according to him the effects of LF in experimental models were variant and dependent on an individual PBMC reactivity, mitogen or alloantigen and LF concentration, and that results suggest that the differential action of LF might be due to its ability to sense the activation status of lymphocyte. So our current results agrees also with Takakura et al., (2004) he reported that a role of LF in the increased recruitment of neutrophils in blood, thus protecting mice from bacteremia also showed that alleviation of oral candidiasis by LF feeding to mice is correlated with the enhancement of the number of leukocytes and their cytokine responses in regional lymph nodes against candida infection. And Wakabayashi et al., (2003) who recorded an increased number of natural killer (NK) cells, increased phagocytosis - enhancing effect, and an increased recruitment of neutrophils in blood and modulation of myelopoiesis made by LF.

This result of leukocytes counts was accompanied by cytokine IL-10 changes within the different groups; while rats treated with LF (group 2) in comparison to control group (group 1), and rats treated with LF after intoxication with DZN (group 4), rats treated with LF as prevention to the toxicity of DZN (group 5) in the comparison to the DZN group (group 3) showed significant increase in IL-10 at the three collections $\left(5^{\text {th }}, 6^{\text {th }}\right.$, and $7^{\text {th }}$ week), rats intoxicated with DZN (group 3) showed significant decrease in IL-10 at the three collections $\left(5^{\text {th }}, 6^{\text {th }}\right.$, and $7^{\text {th }}$ week).

The current results express clearly the effect of the DZN as an oxidative stress inducer and the antioxidant effect of LF which agrees with Guillen et al., (2002) who recorded that an up regulation of antiinflammatory IL-4 and IL-10 was found after LF administration in rats with colitis. Also agrees with Togawa et al. (2002) who reported that Bovine LF regulates cytokines production by splenocytes of obstructive jaundiced rats. In addition, LF enhances the secretion of the anti-inflammatory cytokines IL-10 and IL-4, and reduces colitis in rats.

Changes of albumin and globulin levels give an early, trustee diagnostic information and prognosis, so Protein electrophoresis is a considerable conventional asserted technique to estimate the components of protein of serum plasma in human and animal. In the current study rats treated with DZN showed significant decrease in total proteins (TP) through the three collections $\left(5^{\text {th }}, 6^{\text {th }}\right.$, and $7^{\text {th }}$ weeks), accompanied by increased $\mathrm{A} / \mathrm{G}$ ratio 
at the first and second collections $\left(5^{\text {th }}\right.$ and $6^{\text {th }}$ weeks), significant decrease in globulin through the three collections $\left(5^{\text {th }}, 6^{\text {th }}\right.$, and $7^{\text {th }}$ weeks), accompanied by significant albumin level decrease in the third collection, thus according to these result of this study we suggest that DZN induced liver damage and dysfunction, which agrees with Chatterjea and Shinde (2005) they recorded a noticed increase in aminotransferase levels of (ALT and AST) and the level of ALP as well as the decrease in the levels of total protein and albumin in the serum, as the major diagnostic symptoms of liver diseases. And also agrees with Mossa et al (2011); Gomes et al., (1999); Srivastava and Raizada (1999) they showed that OPIs (e.g., diazinon) caused an increase in activities of ALP, ALT and AST enzymes. And disagrees with Mansour et al., (2010) who reported that the increase of total protein and $\mathrm{A} / \mathrm{G}$ ratio in DZN -treated groups may be due to liver dysfunctions and high elevation of the plasma enzymes, and Abdel-Tawab et al., (2012) who recorded an increased levels of total proteins and decreased levels of $\mathrm{A} / \mathrm{G}$ raio in rats intoxicated with DZN.

In the current study rats treated with LF showed significant increase in total proteins $\mathrm{TP}$ at the second collection only ( $6^{\text {th }}$ weeks), accompanied by decreased $\mathrm{A} / \mathrm{G}$ ratio at the first collection $\left(5^{\text {th }}\right.$ weeks), and increased $A / G$ ratio at the third collection ( $7^{\text {th }}$ weeks), significant increased globulin levels at the first collection $\left(5^{\text {th }}\right.$ weeks) and decreased levels at the third collection ( $7^{\text {th }}$ weeks), accompanied by significant increased albumin level at the third collection. (Group 4) showed significant elevation in TP, albumin, and globulin at the second and third collections $\left(6^{\text {th }}\right.$, and $7^{\text {th }}$ weeks), (group 5) revealed significant elevation in TP, albumin, and globulin at the three collections $\left(5^{\text {th }}, 6^{\text {th }}\right.$, and $7^{\text {th }}$ week). These findings may give us an indication of the destructive effect of DZN and the modulator property of LF, both groups (group 4 and group 5) showed significant increase in $\mathrm{A} / \mathrm{G}$ ratio at the Third collection $\left(7^{\text {th }}\right.$ week). LF possess an immunomodulator and protective role in liver injury induced by DZN This agree with a study done by Akihio et al., (2009) who recorded a decrease in ALT and AST after treatments with different levels of PEGylated LF and native LF in rats intoxicated with CCL4.

Serum protein or peaks visualized by electrophoresis include albumin and $\alpha 1, \alpha 2, \beta$, and $\gamma$ globulins, among the globulins $\alpha 1$ and a2 include many of the diagnostically important acute phase proteins (for example, $\alpha 2$ macroglobulin, haptoglobulin), whereas $\beta$ globulin include other acute phase proteins in addition to complement and various proteins important for coagulation. The $\gamma$ globulin include the immunoglobulins ( $\operatorname{Ig} \mathrm{A}, \operatorname{IgM}, \operatorname{IgE}$, and $\operatorname{IgG})$, in this study rats treated with DZN (group 3) showed significant decrease in $\gamma$ globulins through the three collections $\left(5^{\text {th }}\right.$, $6^{\text {th }}$, and $7^{\text {th }}$ weeks), at the same time rats treated with Lactoferrin (group 2) showed significant increase in $\gamma$ globulins through the three collections $\left(5^{\text {th }}, 6^{\text {th }}\right.$, and $7^{\text {th }}$ weeks $), \alpha 1$, $\alpha 2$, and $\beta$ globulins did not show a stable result that we can build any suggestion concerning them.

For the immune influence and protective effect of LF we concluded from this study, we found that many other studies are concordant with it; Kruzel et al., (2002); Lee et a., (1998); Zimecki et al., (2004) they suggested that LF has an anti-inflammatory effect that down regulates the immune response prevents septic shock and mortality, Actor et al.,(2009)mention that LF also plays as a promoter of the immune system. LF promotes the maturation of T-cell precursors into competent $\mathrm{T}$ helper cells and stimulates the differentiation of immature $\mathrm{B}$ cells into functional antigen presentation cells. And 
with recent studies done by Chih-Ching et al., (2011) on the effect of porcine LF (pLF) on avian immunity are in agreement with these findings. Recombinant pLF was used as a feed stuff additive in chickens vaccinated for the infectious bursal disease (IBD), a worldwide viral disease of chicken with high mortality. A high dose of pLF leads to significant increases in serum $\operatorname{IgA}, \operatorname{IgG}$ and IBD-specific anti-bodies. This may explain the increase of globulin especially gamma globulins in rats treated with LF in our study. In conclusion, Respond of immune system to lactoferrin influence depends on host individual PBMC reactivity, mitogen or alloantigen and LF concentration, thus LF can play as a good immune modulator and can help in tissue recovery. Use of LF as prevention gives better result than using it as treatment drug. More studies should by committed on LF and through assessment of serum cytokines and protein electrophoresis.

\section{REFERENCES}

Actor JK, Hwang SA, Kruzel ML, (2009). Lactoferrin as a nat- ural immune modulator.Curr Pharm Des 15:19561973

Akihiko SUGIYAMA, Atsushi SATO, Hirohiko SHIMIZU, Kunio ANDOand Takashi TAKEUCHI, (2010). pegylatedlactoferrin enhances its hepatoprotective effects on acute liver injury induced by D-Galactosamine and Lipopolysaccharide in Rats. J. Vet. Med. Sci. 72(2): 173-180

Chatterjea MN, Shinde R, (2005). Text Book of Medical Biochemistry, 6th ed. Jaypee Broth; New-Delhi; p.644.

Chih-Ching Yen, Chih-JieShen, Wu-Huei Hsu, Yi-Hsin Chang, Hsin-Tang Lin, Hsiao-Ling Chen, Chuan-Mu Chen,
(2011). Lactoferrin: an iron-binding antimicrobial protein against Escherichia coli Infection Biometals 24:585-594. DOI 10.1007/s10534-0119423-8

Crouch, S. P., Slater, K. J., and Fletcher, J. (1992). Regulation of cytokine release from mononuclear cells by the ironbinding protein lactoferrin. Blood 80: 235-240.

Farnaud S., Evans R., (2003). Lactoferrin-a multifunctional protein with antimicrobial properties. MolImmunol ;40(7):395-405.

Garfitt, S.J., Jones, K., Mason, H.J., Cocker, J., (2002). Exposure to the organophosphate diazinon: data from a human volunteer study with oral and dermal doses. Toxicol. Lett. 134, 105113.

Garg U.K., Pal A.K., Jha G.J., \&Jadhao S.B., (2004). Pathophysiological effects of chronic toxicity with synthetic pyrethroid, organophosphate and chlorinated pesticides on bone health of broiler chicks.Toxicol.Pathol. 32 (3): $\quad 364-369 . \quad$ DOI: 10.1080/01926230490431745.

Gomes J, Dawodu AH, Lloyd O, Revitt DM, Anilal SV, (1999). Hepatic injury and disturbed amino acid metabolism in mice following prolonged exposure to organophosphorus pesticides. Hum. Exp. Toxicol; 18: 33-7.

Guillen C., McInnes I. B., Vaughan D.M., Kommajosyula S., Van Berkel P. H., Leung B. P., (2002). Enhanced Th1 response to Staphylococcus aureus infection in human lactoferrintransgenic mice. J. Immunol. 168: 3950-3957. 
Hassan, G. A., Salem, M. H., Abd-Allah, G. A., Shakere, N., Abo-Elezz, Z., (1988). Effect of organophosphorus (dimethoate) and pyrethroid (decamethrin) pesticides on plasma levels of cortisol and thyroxine, and on some haematological characteristics in growing male rabbits. Indian J. Anim. Sci. 58, 1395-1401

Haversan L., Ohlsson B. G., Hahn-Zoric M., Hanson L. A., and MattsbyBaltzer I., (2002). Lactoferrin down-regulates the LPS induced cytokine production in monocyte cells via NF-KappaB. Cell.Immunol. 220: 83-95.

Kalender Y. Yel M. KalenderS. (2005). Doxorubicin hepatotoxicity and hepatic free radical metabolism in rats: the effect of vitamin $\mathrm{E}$ and cat- echin, Toxicology 209 39-45.

Kalender Y, Uzunhisarcikli M, Ogutcu A, Acikgoz F, and Kalender S, (2006). Effects of diazinon on pseudocholinesterase activity and haematological indices in rats: the protective role of vitamin E, Environ. Toxicol. Pharmacol. 22 (1):46 -5

Keyser J. W. and Watkins G. L. (1972) Estimation of serum proteins by electrophoresis on cellulose acetate.Vol. 18 no. 12 1541- 1542.

Knapp Michael S. (1996). Methodological issue in evaluating integrated services initiatives. First published. https://doi.org/10.1002/ev.1025

Kruzel M. L., Harari Y., Mailman D., Actor J. K., and Zimecki M., (2002).Differential effects of prophylactic, concurrent and therapeutic lactoferrin treatment on LPS-induced inflammatory responses in mice.Clin. Exp. Immunol. 130: 2531.

Lee WJ, Farmer JL, Hilty M, Kim YB, (1998). The protective effects of lactoferrin feeding against endotoxin lethal shock in germ free piglets. Infect Immunol; 66(4):1421-6.

Mossa AH, Refaie AA, Ramadan A, (2011). Effect of exposure to mixture of four organophosphate insecticides at No Observed Adverse Effect Level (NOAEL) dose on rat liver: the protective role of vitamin C. Res. J. Envir. Toxicol; 5: 323-5.

Ogutcu A, Uzunhisarcikli M, Kalender S, Durak D, Bayrakdar F, Kalender Y, (2006). The effects of organophosphate insecticide diazinon on malondialdehyde levels and myocardial cells in rat heart tissue and protective role of vitamin E, Pesticide Biochem. Physiol. 86 93-98.

Srivastava MK, Raizada RB, (1999). Assessment of the no observed- effect level (NOEL) of quinalphos in pregnant rats. Food Chem.Toxicol; 37: 649-3.

Takakura N., Wakabayashi H., Ishibashi H., Yamauchi K., Teraguchi S., Tamura Y., et al., (2004). Effect of orally administered bovine lactoferrin on the immune response in the oral candidiasis murine model. J Med Microbiol;53(Pt 6):495-500.

Togawa J., Nagase H., Tanaka K., Inamori M., Nakajima A., Ueno N., Saito T. and Sekihara, H.., (2002). Oral administration of lactoferrin reduces colitis in rats via modulation of the immune system and correction of cytokine imbalance. J 
GastroenterolHepatol; 17 (12): 12911298.

Wakabayashi H., Takase M., and Tomita M., (2003). Lactoferrin derived from milk protein

Ward P. P., and Conneely O. M., (2004). Lactoferrin: role in iron homeostasis and host defense against microbial infection. Biometals 17: 203- 208.

Weichselbaum TE. (1964). An accurate and rapid method for the determination of proteins in small amounts of blood serum and plasma. Am J Clin. Pathol. 10:40-9.

Zimecki M, Wlaszczyk A, Zagulski T, Kubler A, (1998). Lactoferrin lowers serum interleukin 6 and tumor necrosis factor alpha levels in mice subjected to surgery. Archivum Immunologiaeet Therapiae Experimentalis (Warsz) 46 (2), 97- 104,

Zimecki M, Dariusz S, Pniak A. S, and Marian L. K, (2001). Lactoferrin Regulates Proliferative Response of Human Peripheral Blood Mononuclear cells to phytohaemaglutinin and Mixed Lymphocyte Reaction. Archivum Immunologiaeet Therapiae Experimentalis, 49, 147- 154.

Zimecki M, Spiegel K, Wlaszczyk A, Kubler A, Kruzel M, (1999). Lactoferrin increases the output of neutrophil precursors and attenuates the spontaneous production of TNF-alpha and IL-6 by peripheral blood cells. Arch Immunol Ther Exp (Warsz);47(2):113-8 